\title{
PREVENÇÃO DO CÂNCER DE COLO UTERINO NA CONCEPÇÃO DA POPULAÇÃO FEMININA DE UMA CIDADE DO INTERIOR DO ESTADO DE SÃO PAULO
}

Fabiola Azevedo Mello, Leonilda Chiare Galle, Rosana Leal do Prado

Universidade do Oeste Paulista - UNOESTE, Curso de Biomedicina, Presidente Prudente, SP. E-mail: leonilda@unoeste.br

\section{RESUMO}

Apesar dos programas de prevenção, o câncer de colo uterino (CCU) continua sendo grande preocupação para a saúde pública. Este estudo foi proposto para conhecer as dificuldades que interferem na cobertura do exame de Papanicolau em uma população feminina. Trata-se de um estudo do tipo observacional, primário, prospectivo, transversal e quantitativo. Houve associação estatística significativa entre a realização anual do exame e o estado civil, pois $84,13 \%$ das mulheres que realizam o exame anualmente são casadas, a maioria declarou estar empregada, apenas 33,07\% são usuárias do Sistema Único de Saúde (SUS) e 16,28\% não participam do programa de rastreamento com regularidade. A vergonha foi a justificativa mais apresentada para a não realização do exame, seguida pela falta de tempo, não confiar no SUS e julgar o exame desnecessário. Conclui-se que estratégias educativas são fundamentais para a prevenção do CCU, na população estudada, sendo que, mulheres solteiras, desempregadas e mais jovens necessitam maior atenção.

Palavras-chave: Câncer do colo uterino, exame de papanicolau, prevenção, conhecimento, população feminina.

\section{PREVENTION OF CERVICAL CANCER IN THE CONCEPTION OF THE FEMALE POPULATION OF A CITY INLAND OF SÃO PAULO STATE.}

\begin{abstract}
Despite of prevention programs, the cervical cancer (CC) continues being a great concern to the public health service. This study was proposed to find the difficulties that interfere at the coverage of the Pap smear in a female population. This is an observational study, primary, prospective, cross-sectional and quantitative. It was observed a statistic significance in the association between the annual exam execution and the marital status, where $84.13 \%$ of the women who performed the annual exam was married, the majority declared to be employed, only 33.07\% are Sistema Único de Saúde (SUS) users and $16.28 \%$ doesn't performed the regularly screening program. The shame was the major justification of the non perform of exam, followed by the lack of time, non confidence at SUS service's and judge the exam unnecessary. It was concluded that educational strategies are fundamental for the prevention of the CC in the population studied, and that single, unemployed and younger women need more attention.
\end{abstract}

Keywords: Cervical cancer, pap smear, prevention, knowledge, female population.

\section{INTRODUÇÃO}

O câncer de colo uterino (CCU), também chamado de cervical, é uma doença de evolução lenta e apesar dos programas de prevenção, continua sendo de grande preocupação para a saúde pública. Há anos esse tipo de câncer destaca-se devido as altas taxas de morbidade e mortalidade entre a população feminina, com grande destaque nos países em desenvolvimento ${ }^{1}$.

Segundo o Instituto Nacional do Câncer (INCA), é o tumor mais frequente na população feminina e a quarta causa de morte de mulheres por câncer no Brasil. Em 2014, 5.160 mulheres morreram por causa da doença, sendo este, o tipo de câncer que apresenta maior potencial de 
prevenção, próximo de $100 \%^{2}$. O início precoce das relações sexuais, número de parceiros sexuais, multiparidade, antecedentes de doenças venéreas, baixa escolaridade, uso de anticoncepcional oral por tempo prolongado e tabagismo são fatores de risco para o desenvolvimento da doença ${ }^{3}$.

Ultimamente a infecção persistente pelo vírus papiloma humano (HPV) tem sido demonstrada em várias pesquisas como fator importante e está presente em mais de $90 \%$ dos casos $^{4}$. A maioria das lesões é de evolução lenta e os estágios precursores em estado não-invasivo podem ser detectados no exame citológico, que é oferecido pelo programa de rastreamento deste tipo de câncer no Brasil, e mesmo assim a taxa de mortalidade continua alta ${ }^{5}$.

A tecnologia para o rastreamento das lesões precursoras do CCU há anos é realizada com base nas alterações celulares observadas em esfregaços cervicais proposto por George Papanicolaou, o método é de baixo custo, eficaz, adotado mundialmente para reduzir o desenvolvimento do câncer uterino e aplicado com protocolos bem definidos em programas de prevenção do $\mathrm{CCU}^{3,5-9}$.

O tratamento se fundamenta no diagnóstico, estadiamento e prognóstico da doença, tendo como parâmetro a avaliação da localização, tamanho e tipo histológico do tumor, a idade e as condições de saúde da mulher. No estadiamento inicial da doença é utilizada a cirurgia para remoção completa do tumor e a associação da radioterapia e/ou quimioterapia é decidida com base no estágio da doença e nas características tumorais $^{10}$.

Supõe-se que a exposição das partes íntimas e a posição ginecológica exigidas para a realização do exame, o toque ginecológico, a introdução do espéculo, da espátula e da escova para coleta via vaginal estão entre os fatores que esquivam as mulheres da realização do exame. Julga-se ser inevitável que as mulheres só darão importância ao programa de prevenção ao câncer ginecológico quando as mesmas tiverem compreensão de sua necessidade e importância.

O objetivo do estudo foi identificar o conhecimento e as dificuldades que interferem na cobertura do exame de Papanicolaou na população feminina de uma cidade do interior do estado de São Paulo.

\section{METODOLOGIA}

Trata-se de um estudo do tipo observacional, primário, prospectivo, transversal e quantitativo, submetido e aprovado pelo Comitê de Ética em Pesquisa (CEP) da Univerdade do Oeste Paulista (UNOESTE), sob o número $n^{\circ}$ 1510 e que cumpriu os termos da Resolução 466/2012 do Conselho Nacional de Saúde.

Segundo os resultados do Censo 2010, a população feminina do município em estudo, acima de 10 anos de idade, foi representada por $2.064(40,9 \%)$ mulheres do total de $5.039^{11}$, resultando no cálculo amostral de 251 mulheres.

As participantes desta pesquisa foram selecionadas de maneira aleatória e contatadas pessoalmente pelo pesquisador. Foi estabelecido que na falta do consentimento livre e esclarecido ou na desistência da participação em qualquer momento a entrevistada seria excluída da pesquisa.

O local de contato com as mulheres foi em suas residências a partir da rua principal, tomando-se a primeira residência como uma unidade de coleta de dados. A sequência foi estabelecida de tal forma que entre as unidades subsequentes de residências, duas não seriam abordadas, mesmo quando na última residência visitada não houvesse residente que preenchesse os critérios de participação. Somente em caso de recusa de participação ou na ausência da moradora a residência seria substituída pela próxima e se a situação se repetisse a residência anterior seria a escolhida. 0 procedimento foi o mesmo para todas as ruas paralelas e a abordagem foi encerrada, quando o número de 251 entrevistadas foi atingido.

O questionário aplicado abordou: idade (a parti de 10 anos), estado civil, tipo de serviço utilizado para coleta do preventivo, escolaridade, ocupação, frequência da realização do exame e fatores que julgam serem de risco para o desenvolvimento da doença, sendo baseado nas orientações do INCA ${ }^{12}$.

A avaliação do conhecimento, sobre os fatores de risco para o desenvolvimento do $\mathrm{CCU}$, foi adaptada de $\operatorname{Araujo}^{13}$ e, cada resposta correta foi somado um ponto. O conhecimento foi categorizado como alto, quando a entrevistada somou mais de 11 pontos, médio entre 6 e 10 e baixo até 5 pontos.

Para as análises descritivas e testes de associação das variáveis, os resultados foram registrados em planilhas e a análise estatística 
foi realizada por meio do teste de qui-quadrado $\left(X^{2}\right)$, com significância estatística de $p \leq 0,05$.

\section{RESULTADOS}

No período de junho de 2012 a fevereiro de 2013 em um município do interior do Estado de São Paulo, foram entrevistadas 251 mulheres, todas com idade a partir de 18 anos. A Figura 1 apresenta a distribuição das entrevistadas de acordo com a faixa etária, sendo que a maioria estava na faixa etária de 18 a 59 anos $(90,84 \%)$.

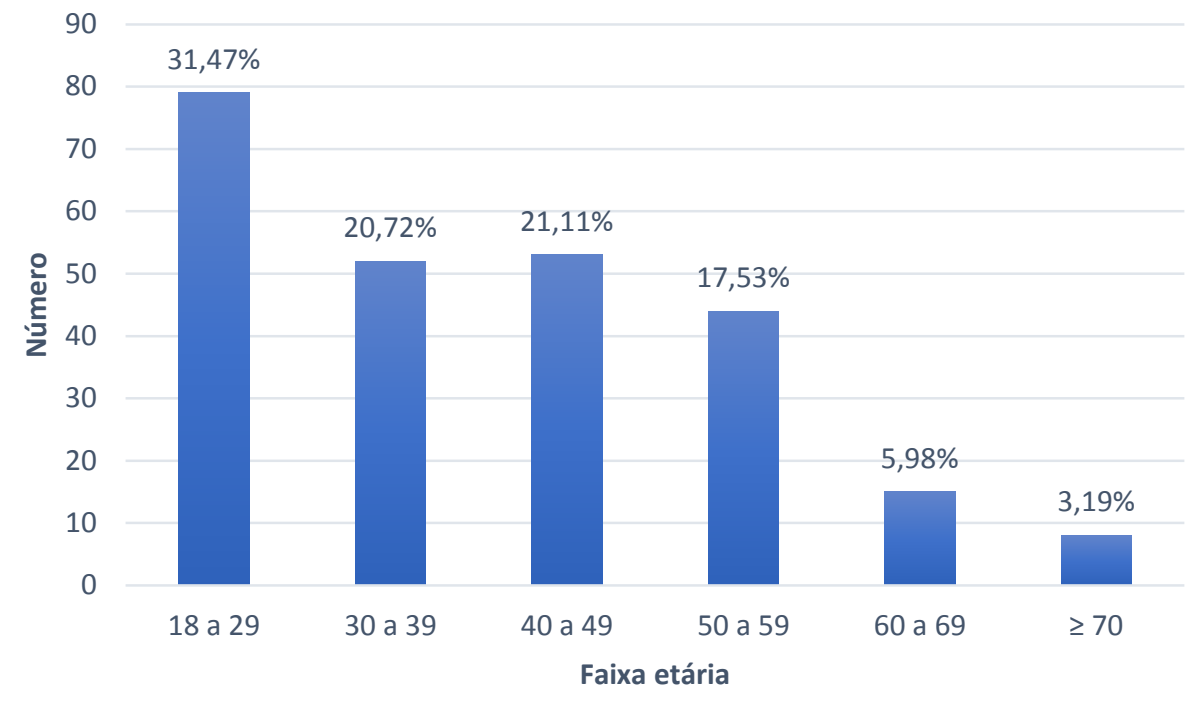

Figura 1. Distribuição percentual das 251 mulheres entrevistadas, de acordo com a faixa etária.

Sobre o serviço de saúde, 133 (52,99\%) afirmaram utilizar o Sistema Ùnico de Saúde (SUS), 104 (41,43\%) afirmam utilizar outros serviços (particular, por exemplo) e 14 (5,58\%) não informaram o tipo de serviço que utilizam.
Na Tabela 1, pode-se observar a distribuição percentual de mulheres de acordo com a caracterização sociodemográfica.

Tabela 1. Distribuição percentual de acordo com a caracterização sóciodemográfica das mulheres entrevistadas.

\begin{tabular}{lcc}
\hline Estado civil & $\mathrm{N}$ & $\%$ \\
\hline Casada & 145 & 57,77 \\
Solteira & 90 & 35,86 \\
Viúva & 16 & 6,37 \\
\hline Escolaridade & & \\
\hline Ensino fundamental & 59 & 23,51 \\
Ensino médio & 109 & 43,43 \\
Ensino superior & 74 & 29,48 \\
lletrado & 9 & 3,58 \\
\hline Ocupação & & \\
\hline Empregada & 141 & 56,18 \\
Desempregada & 80 & 31,87 \\
Estudante & 29 & 11,55 \\
Sem resposta & 1 & 0,40 \\
\hline
\end{tabular}

A pesquisa sobre a realização do exame de Papanicolau para a prevenção do câncer de colo uterino por esta população revelou que 174 $(69,33 \%)$ realizam o exame com frequência anual e $21(8,37 \%)$ a cada 2 anos, $16(6,37 \%)$ realizam entre 3 e 5 anos, $12(4,78 \%)$ mais que 5 anos e 28 $(11,15 \%)$ afirmaram que nunca realizaram o exame. 
Sobre a frequência de realização do exame e sua relação com o estado civil (Tabela 2), foi possível observar que a maioria das mulheres que realizam o exame anualmente era casada. $\mathrm{A}$ frequência de realização do exame mostrou associação estatística significante com o estado civil $(p<0,0001)$, por meio do teste de quiquadrado $\left(X^{2}\right)$.

Tabela 2. Distribuição da frequência de realização do exame de Papanicolau em relação ao estado civil das 251 mulheres entrevistadas.

\begin{tabular}{lcccccc} 
& \multicolumn{2}{c}{ Casada } & \multicolumn{2}{c}{ Solteira/Viúva } & \multicolumn{2}{c}{ Total } \\
Frequência & $\mathbf{n}$ & $\mathbf{\%}$ & $\mathbf{n}$ & $\mathbf{\%}$ & $\mathbf{n}$ & $\boldsymbol{\%}$ \\
\hline Anual & $122^{*}$ & 84,13 & 52 & 49,05 & 174 & 69,32 \\
2 anos & 7 & 4,83 & 14 & 13,21 & 21 & 8,37 \\
$3-5$ anos & 8 & 5,51 & 8 & 7,55 & 16 & 6,37 \\
mais 5 anos & 7 & 4,83 & 5 & 4,71 & 12 & 4,78 \\
Nunca & 1 & 0,69 & 27 & 25,47 & 28 & 11,16 \\
Total & 145 & 100 & 106 & 100 & 251 & 100 \\
\hline
\end{tabular}

${ }^{*} p<0,0001 ; X^{2}=50,121$

Ressalta-se que $28 \quad(11,16 \%)$ informaram que nunca fizeram o exame e entre estas, apenas uma afirmou ser casada, sendo as demais solteiras ou viúvas.

Entre as 174 mulheres que realizam o exame anualmente, 91 (52,30\%) afirmam que utilizam outros serviços para a realização do exame preventivo e $83(47,70 \%)$ afirmaram que utilizam o Sistema Único de Saúde (SUS). A frequência de realização do exame e o tipo de serviço que utilizam para a coleta estão distribuídos na Tabela 3.

Tabela 3. Distribuição da frequência de realização do exame de Papanicolau em relação ao serviço de saúde utilizado entre as 251 mulheres entrevistadas.

\begin{tabular}{lcccc}
\hline & \multicolumn{2}{c}{ Utilizam o SUS } & \multicolumn{2}{c}{ Outros } \\
\hline Frequência & $\mathbf{n}$ & $\mathbf{\%}$ & $\mathbf{n}$ & $\mathbf{\%}$ \\
\hline Anual & 83 & 69,17 & 91 & 88,35 \\
2 anos & 15 & 12,5 & 6 & 5,83 \\
3-5 anos & 13 & 10,83 & 3 & 2,91 \\
mais 5 anos & 9 & 7,5 & 3 & 2,91 \\
Total & 120 & 100 & 103 & 100 \\
\hline
\end{tabular}

As mulheres que não realizam o exame anualmente ou a cada 2 anos foram questionadas sobre as dificuldades encontradas para não atender esta recomendação. A justificativa mais frequente foi vergonha, mencionada 17 vezes, seguida pela falta de tempo, mencionada 12 vezes. Nove mulheres informaram que não confiam no serviço do SUS e 6 julgam que o exame é desnecessário. As demais mulheres relatam descuido, falta de acesso e de vontade, relaxo e medo.
Da mesma forma, os dados obtidos da frequência de realização do exame foram distribuídos com relação à ocupação e apresentados na Tabela 4, revelando que a maioria das entrevistadas que realizam o exame anualmente são empregadas. A variável ocupação e frequência de realização do exame mostraram associação estatística significativa $(p<0,05)$. 
Tabela 4. Distribuição da frequência de realização do exame de Papanicolau em relação a ocupação das 251 mulheres entrevistadas.

\begin{tabular}{lcccc}
\hline Frequência & Empregada & Estudante & Desempregada \\
\hline Anual & $105^{*}$ & 14 & 55 \\
2 anos & 9 & 4 & 7 \\
3-5 anos & 9 & 0 & 7 \\
mais 5 anos & 7 & 1 & 4 \\
Nunca & 11 & 10 & 7 \\
Total & 141 & 29 & 80 \\
\hline
\end{tabular}

$* p=0,0038 ; X^{2}=22,707$

Observou-se que apenas 11 (4,38\%) das entrevistadas apresentaram alto conhecimento sobre os fatores de risco para o desenvolvimento da doença, $96 \quad(38,25 \%)$ apresentaram conhecimento médio e 142 mulheres (56,57\%) baixo conhecimento. A infeç̧ão pelo Papiloma Vírus Humano (HPV) foi identificada como fator de risco por $112(44,62 \%)$ e o uso de preservativo por 28 (11,15\%) entrevistadas. Em relação à escolaridade das 28 entrevistadas que apontaram o uso do preservativo como fator de risco para o desenvolvimento da lesão, 3 afirmaram ter o ensino superior, 24 fundamental e médio e apenas 1 era iletrada.

\section{DISCUSSÃO}

Neste estudo de abordagem sobre a prevenção do CCU na concepção da população feminina, aproximadamente $91 \%$ das participantes tinham entre 18 e 59 anos, com média de idade de 42 anos. Embora 0 questionário tenha sido oferecido para mulheres mais jovens, não houve nenhuma participante com idade inferior a 18 anos. Aproximadamente $58 \%$ das entrevistadas afirmaram serem casadas e $53 \%$ usuárias do SUS.

A falta de regularidade na coleta do exame de Papanicolau é apontada na literatura como o principal fator que limita a prevenção do $\mathrm{CCU}^{12}$ No Brasil o rastreamento das lesões é realizado através do exame de Papanicolau e preconizado anualmente para as mulheres entre 25 e 60 anos de idade, ou que já tenham iniciado a atividade sexual mesmo antes desta faixa etária. Quando há dois exames anuais consecutivos com resultado negativo, o intervalo da coleta é trienal ${ }^{9,14}$. Apesar do programa de rastreamento do CCU já estar há alguns anos estabelecido no Brasil, a taxa de morte por este tipo de câncer não tem reduzido ${ }^{15}$.

Desta forma, a periodicidade de realização do exame é estabelecida de acordo com variáveis individuais. As mulheres são orientadas a fazer o exame anualmente e recebem a orientação sobre a periodicidade adequada de acordo com a avaliação dos resultados ${ }^{9}$. Apesar da população em estudo ter acesso ao exame no posto de saúde através do SUS, apenas $83(33,07 \%)$ usuárias do SUS afirmam que realizam o exame anualmente, 91 $(36,25 \%)$ o fazem anualmente em outros serviços, outras 12 (4,78\%) mulheres entrevistadas realizaram o exame há mais de cinco anos e $28(11,5 \%)$ nunca realizaram. Desta forma, apenas $40(16,28 \%)$ que não foram atingidas com eficiência pelo programa de rastreamento, revelando uma cobertura de $83,72 \%$ bem próximo ao estimado pela Organização Mundial da Saúda (OMS). Estes achados evidenciam a necessidade de uma intervenção educativa, com o objetivo de adequar a periodicidade da coleta do exame preventivo visando maior efetividade na prevenção do CCU.

Em pesquisa de Vale et al. ${ }^{5}$, o percentual de mulheres que realizam o exame anualmente pelo SUS é inferior aos 50\% das usuárias do SUS na cidade de Amparo.

O Sistema de Informação do Câncer do Colo do Útero e o Sistema de Informação do Câncer de Mama (SISCOLO), utilizado pelo SUS, registram os exames com alterações citológicas realizados no Brasil e controla diversas variáveis, mas não identifica quem está sem controle ou o fez há três anos ou mais ${ }^{17}$. Uma estratégia que inclui o rastreamento individual, o conhecimento dos obstáculos e das dificuldades de acesso de algumas mulheres ao serviço de saúde poderia ampliar a cobertura da coleta do exame e seria ainda mais efetivo quando associado ao recrutamento sistemático ou busca ativa ${ }^{14,16}$.

Embora a orientação para a periodicidade da realização do exame de Papanicolau não dependa do estado civil da mulher, foi possível afirmar que a maioria das mulheres participantes desta pesquisa que faz o 
exame anualmente são casadas. Segundo estudo de Melo et al. ${ }^{17}$, entre as mulheres com alterações citopatológicas, os fatores de risco para o CCU esteve mais presente entre as mulheres casadas, já para Costa et al. ${ }^{4}$ não há associação entre lesão do colo uterino e estado civil, porém há consenso entre vários autores que as lesões dependem do grau de imunidade das Doenças Sexualmente Transmissíveis (DSTs) e do número de parceiros sexuais ${ }^{1,17}$.

Quanto à ocupação, a maioria das mulheres que colhem o exame anualmente afirma que estão empregadas. É possível que as mulheres casadas e as que trabalham tenham mais cuidado com a saúde ou mais acesso à informação de saúde, principalmente preventiva, contrariando nossas expectativas, já que esperávamos que as mulheres empregadas tivessem menos tempo de procurar o serviço de saúde para a realização do preventivo e consequentemente, não $o$ realizariam periodicamente.

A justificativa mais frequente para a não realização do exame foi vergonha, mencionada por 17 mulheres, seguida pela falta de tempo mencionada por 12, outras 9 informaram que não confiam no serviço do SUS e 6 julgam que o exame é desnecessário. As demais mulheres relatam descuido, falta de acesso e vontade, relaxo e medo. Nossos resultados corroboram com várias pesquisas que apontam o baixo nível socioeconômico, a baixa escolaridade, vergonha, medo do resultado, dificuldade de deslocamento, tensões emocionais provocadas pelo procedimento e o tempo gasto com a coleta, sendo as dificuldades mais apresentadas pelas mulheres por ocasião da coleta do exame. Estes sentimentos não devem ser ignorados, ao contrário, os serviços de saúde envolvidos no programa de prevenção do CCU, devem buscar formas para que esses aspectos sejam minimizados e trabalhados juntos com as medidas educativas sobre a importância do exame $^{9,14-16}$. 0 início precoce das relações sexuais, número de parceiros sexuais, multiparidade, antecedentes de doenças venéreas, baixa escolaridade, uso de anticoncepcional oral por tempo prolongado e tabagismo são fatores de risco para o desenvolvimento da doença. Ultimamente a infecção por HPV é demonstrada em várias pesquisas como fator importante e está presente em mais de $90 \%$ dos casos de câncer cervical $^{16}$. Costa et al. ${ }^{4}$ relatam que a presença das lesões celulares estão significativamente associadas ao número de parceiros, a história de DST e tabagismo, já que o epitélio cervical das fumantes tem o número de células de Langerhans diminuído em relação as que não fumam, facilitando as lesões virais. Outras pesquisas apontam que a frequência de lesões precursoras do câncer do colo uterino ocorre em mulheres com a faixa etária inferior a esperada, principalmente adolescentes que aparentam estar mais predispostas aos riscos associados ao câncer uterino $0^{4,14,18,19}$.

A análise sobre o conhecimento dos fatores de risco para o desenvolvimento do CCU pelas mulheres entrevistadas, segundo as categorias estabelecidas para esta pesquisa, revelou que 142 mulheres $(56,57 \%)$ apresentam baixo conhecimento, apesar da infecção pelo HPV ser sido apontada como a causa da doença por $122(44,62 \%)$ das entrevistadas e apenas 11 mulheres (4,38\%) apresentam alto conhecimento. Por outro lado, 28 (11,15\%) mulheres, principalmente jovens, entre 18 e 29 anos, mencionaram o uso do preservativo como um fator de risco para o desenvolvimento da doença, ao contrário de prevenir DSTs, o que mostrou a falta de informação sobre a prevenção em uma parcela da população. De acordo com Ferreira ${ }^{18}$, devido à alta incidência de DSTs, principalmente o HPV, que muitas vezes consiste em infecções subclínicas ou latentes, o uso de preservativo em todas as relações sexuais vem se mostrando a forma mais viável de prevenção, mesmo para aquelas que tem um único parceiro sexual. De acordo com o estudo feito por Melo et al. ${ }^{17}$ é necessário ampliar a informação gerando mais conhecimento à população sobre o exame preventivo e reforçar os fatores que levam ao desenvolvimento das DSTs e CCU.

\section{CONCLUSÃO}

Acreditamos ser a educação em saúde uma forte aliada da prevenção de doenças, contribuindo para a formação da consciência crítica, estimulando a mudança do comportamento e a organização individual e coletiva, quando consegue promover a adoção de novos hábitos e condutas de saúde.

Atingir as pessoas com a informação é um grande desafio, mas é uma prática necessária que deve ser aliada a outras estratégias para facilitar a disseminação da informação de forma criativa e atraente a todas as mulheres e nesta população de forma mais intensa as solteiras, desempregadas e mais jovens. 
Para a cobertura adequada do exame preventivo do CCU é necessário identificar e orientar individualmente as mulheres que estão sem controle ou que não fazem o exame regularmente de acordo com o programa SISCOLO, além de estabelecer estratégias educativas para a conscientização da população sobre os fatores de risco para o desenvolvimento da doença.

Apesar de a maioria das entrevistadas, nesta pesquisa apresentarem baixo conhecimento sobre os fatores de risco para o desenvolvimento da doença, o rastreamento das lesões através do exame de Papanicoloau foi aproximado à cobertura estabelecida pela OMS.

\section{AGRADECIMENTOS}

Agradecemos a todas as participantes da pesquisa.

\section{DECLARAÇÃO DE CONFLITO DE INTERESSE}

Os autores declaram não haver qualquer conflito de interesse que possa interferir na imparcialidade deste trabalho científico.

\section{REFERÊNCIAS}

1. Pinho AA, Franca-Junior I. Prevenção do câncer de colo do útero: um modelo teórico para analisar o acesso e a utilização do teste de Papanicolaou. Rev Bras. Saúde Mater Infant. 2003;3(1):95-112. DOI:

https://doi.org/10.1590/S151938292003000100012

2. Instituto Nacional do Câncer. Prevenção do Câncer de Colo Uterino no Brasil. INCA, 2015. Acesso em: 15 jun 2015. Disponível em: http://www2.inca.gov.br/wps/wcm/connect/tipo sdecancer/site/home/colo utero/deteccao prec oce

3. Aleixo Neto A. Epidemiological aspects of cervical neoplams. Rev Saúde Pública. 1991;25(4):326-33. DOI: https://doi.org/10.1590/S0034-

\section{3}

4. Costa OLN, Guimarães MH, Júnior OSL, Leal EAS, Nascimento TL, Vitoriano MN. Cervical cancer precursor lesions in adolescent and young adult women of Rio Branco - Acre. Rev Bras Ginecol Obstet. 2003;25(2):81-6.

5. Vale DBAP, Morais SS, Pimenta AL, Zeferino LC. Avaliação do rastreamento do Câncer do colo Uterino na Estratégia Saúde da Família no Município de Amparo, São Paulo, Brasil. Cad
Saúde Pública. 2012;26(2):383-90. DOI: http://dx.doi.org/10.1590/S0102311X201000020017

6. Borges MFSO, Dotto LM, Koifman RJ, Cunha MA, Muniz PT. Prevalence of uterine cervical cancer testing in Rio Branco, Acre State, Brazil, and factors associated with non-participation in screening. Cad Saúde Pública. 2012;28(6):115666. DOI: http://dx.doi.org/10.1590/S0102311X2012000600014

7. Eluf-Neto J, Nascimento CM. Cervical cancer in Latin America. Semin Oncol. 2001;28(2):188-97. DOI: https://doi.org/10.1053/sonc.2001.21966

8. Nomenclatura Brasileira para Laudos Cervicais e Condutas Preconizadas. Ministério da Saúde. 2.ed. Rio de Janeiro: Ministério da Saúde; 2006.

9. Instituto Nacional de Câncer. Periodicidade de realização do exame preventivo do câncer do colo do útero: normas e recomendações do INCA. Rev Bras Cancerol. 2002;48(1):13-15. Acesso em: 21 fev. 2015. Disponível em: http://www.inca.gov.br/rbc/n 48/v01/pdf/norm as.pdf

10. Frigato $S$, Hoga LAK. Assistência à mulher com câncer de colo uterino: o papel da enfermagem. Rev Bras Cancerologia. 2003;49(4):209-14.

11. IBGE - Instituto Brasileiro de Geografia e Estatística. Acesso em: 01 jul 2015. Disponível em: http://www.ibge.gov.br

12. Instituto Nacional do Câncer. Prevenção do Câncer de Colo Uterino no Brasil. INCA, 2015. Acesso em: 7 jun 2015. Disponível em: http://www2.inca.gov.br/wps/wcm/connect/cbe 1398046d98a238c0ced5120665fa8/Avalia\%C3\%A 7\%C3\%A3o+indicadores+colo+e+mama+2013.pdf ?MOD=AJPERES\&CACHEID=cbe1398046d98a238c Oced5120665fa8

13. Araujo WDB, Almeida MEF, Santos CEM, Pizzido VR. Avaliação do conhecimento de manipuladores de alimentos quanto às boas práticas de fabricação. Acesso em: 28 set 2014. Disponível em: http://www.reitoria.uri.br/ vivencias/Numero_0 09/artigos/artigos_vivencias_09/n9_8.htm

14. Farias LM, Feitoza AR, Moura ADA, Silca SMG. Conhecimento e motivações das mulheres acerca do exame de papanicolaou: subsídios para a prática de enfermagem. Rev Rene Fortaleza. 2010;11(1):94-104. Acesso em: 4 jan 2013. Disponível em: http://www.repositorio.ufc.br/bitstream/riufc/40 13/1/2010_art_adamoura.pdf

15. Martins LFL, Thuler LCS, Valente JG. Coverage of the Pap smear in Brazil and its determining 
factors: a systematic literature review. Rev Bras Ginecol Obstet. 2005;27(8):485-92. DOI: http://dx.doi.org/S0100-72032005000800009

16. Albuquerque KM, Andrade CLT, Aquino EML, Menezes G, Szwarcwald CL. Pap smear coverage and factors associated with non-participation in cervical cancer screening: an analysis of the

Cervical Cancer Prevention Program in Pernambuco State, Brazil. Cad Saúde Pública. 2009;25(2):301-9. DOI: http://dx.doi.org/S0102311X2009001400012

17. Melo SCCS, Prates L, Carvalho MDB, Marcon SS, Pelloso SM. Alterações citopatológicas e fatores de risco para a ocorrência do câncer de colo uterino. Rev Gaúcha Enferm. 2009;30(4):602-8. DOI: http://dx.doi.org/S198314472009000400004

18. Ferreira MLSM. Influence reasons that inhibit women from doing papanicolaou test. Rev Enferm. 2009;13(2):378-8. DOI: http://dx.doi.org/S1414-81452009000200020

19. Hackenhaar AA, Juraci AC, Marcos RD. Pap smears of $20-59$ year-old women in Pelotas, Southern Brazil: prevalence, approach and factors associated with not undergoing the test. Rev Bras Epidemiol. 2006;9(1):103-11. DOI: http://dx.doi.org/S1415-790X2006000100013

Recebido para publicação em 24/07/2015

Revisado em 21/06/2016

Aceito em 24/01/2018 